\title{
JOURNAL OF Dry EYE AND Ocular Surface Disease
}

Original Article

\section{PELLEVÉ ${ }^{T M}$ VS LIPIFLOW ${ }^{\text {TM }}$ MGD-RELATED DRY EYE TREATMENT STUDY: THE THERMALID ${ }^{\text {TM }}$ PROCEDURE}

\section{A Pilot Study Comparing the efficacy of the Pellevé System to LipiFlow for the Treatment of Dry Eye due to Meibomian Gland Dysfunction}

Edward H. Jaccoma, MD, Craig Litherland, MS, Andrew Jaccoma, BS, MBA, Aseef H. Ahmed, BS, MS

University of New England College of Osteopathic Medicine, Biddeford, ME

Correspondence may be directed to: ejaccoma@aol.com

Submitted: January 4, 2018. Accepted: February 28, 2018. Published: March 28, 2018.

\begin{abstract}
Objective

To assess the safety and efficacy of the Pellevé Wrinkle Treatment System as compared to LipiFlow, for treatment of dry eye syndrome due to meibomian gland dysfunction (MGD).

\section{Materials and Methods}

In this split face, randomized study, 10 patients who met specific criteria for inclusion were followed after treatment with the Pellevé Wrinkle Reduction System (what the authors have dubbed the "ThermaLid procedure") and a standard LipiFlow procedure performed the same day, with a 1 and 3 month follow-up. Pre- and post-treatment MGD was assessed using a variety of subjective and objective measurements.
\end{abstract}

\section{Results}

The function of the meibomian glands improved significantly from baseline to 3 months for both ThermaLid and Lipiflow treated eyes, with no significant difference between them, as measured by MGE grading, wax plug scoring, Standard Patient Evaluation of Eye Dryness (SPEED) and Ocular Surface Disease Index (OSDI) scoring. A significant change in conjunctival staining was found after both treatments at the 1-month time point but not the 3 -month time point. The ThermaLid treatment but not the Lipiflow treatment, significantly decreased MARX line measurements at the 3-month time point. Lipiview, Non-Invasive Tear Breakup Time (NITBUT), corneal surface staining, Tear Osmolarity, and anesthetic Schirmer's testing did not show statistically significant improvement, nor differences between treatment modalities.

\section{Conclusions}

ThermaLid treatment of the eyelids appears to show efficacy similar to Lipiflow treatment in reducing symptoms, reducing wax plugging and improving the number of functioning MGs as well as reducing conjunctival staining in MGD-related dry eye disease.

Dry eye disease (DED) is a common chronic, progressive condition that causes eye discomfort, limits vision, interferes with cataract and refractive surgery, and reduces quality of life. ${ }^{1}$ It principally occurs when the tear film is compromised by reduced aqueous tear production and/or excessive tear evaporation. Evaporative dry eye due to meibomian gland dysfunction (MGD) appears to represent the most common DED subtype, ${ }^{2-4}$ although many with MGD lack dry eye symptoms. ${ }^{5}$ 
Dry eye is the most commonly reported reason for seeking medical eye care, and thus dry eye has a significant cost due to direct and indirect health care costs and through reduced productivity at work. ${ }^{6}$ In the United States, the average cost of dry eye management was estimated to be $\$ 11,302$ per sufferer and $\$ 55$ billion overall. ${ }^{7,8}$ With increased life expectancy and an aging population, the economic and social impacts of this condition would be expected to grow substantially. ${ }^{9}$

Baudoin et al describe six separate, inter-related pathophysiologic mechanisms as probably causal to MGD, with obstructive MGD as the most common sub-type leading to evaporative DED. These mechanisms include: primary obstructive hyperkeratinization of the meibomian gland, abnormal meibomian gland secretion, eyelid inflammation, corneal inflammation and damage, microbiological changes, and DED. ${ }^{10,11}$ The heart of obstructive meibomian gland dysfunction (MGD) is the waxy blockage from inspissated secretions relating to hyperkeratinization and an elevated melting point of the solidified oils from stagnation and bacterial flora activity. Current, in-office lid-heating procedural approaches for the treatment of dry eye secondary to obstructive MGD are many. LipiFlow, an automated thermal pulsation system, is currently the only FDA approved treatment, which achieves this by direct heating and massaging of the meibomian glands to express their waxy blockages and restore function. One of the criticisms of alternative methods which employ passive, externally conductive heating of the meibomian glands is that there is an insufficient transfer of heat to attain the critical "wax-melting" needed to unblock the obstructed glands. ${ }^{7}$ To date, there are limited head-to-head comparisons of the efficacy of these alternative technologies in treating MGD and its related DED.

Numerous studies have demonstrated that LipiFlow treatment provides relief for patients from symptoms of MGD including dry eye. Hence, Lipiflow with its electrical conductive heating and vectored massage may be viewed as the current "gold standard" of treating obstructive MGD. ${ }^{12}$

Radiofrequency (RF) electrosurgical systems, first developed in 1928, are the most common energy sources used for cutting and hemostasis in surgery.
Ellman's Pellevé (Wrinkle Reduction - RF) System has been utilized since 2009 to decrease skin laxity and improve rhytids of the face, including the periocular area, by heating the dermis. ${ }^{13,14}$ High-frequency electron flow RF generates heat as a result of the difference in impedance between tissue types, which turns kinetic energy into thermal energy and the surrounding tissues are heated. ${ }^{15}$ This method not only eliminates the problem of heating unwanted target chromophores in the skin, such as melanin, as seen with intense pulsed light, but also allows the heat to be generated in the deep dermis where existing residual collagen bundles are most plentiful. A controlled thermal injury can cause contraction of the thinned collagen in the deep dermis immediately and trigger an inflammatory response that generates new collagen bundle reorganization and thickening evident at 12 weeks as seen on electron microscopy. ${ }^{16,17}$ A controlled thermal contraction down the deep connective tissue bands causes a vertical and three-dimensional tissue contraction, compacting the fatty globules without injuring the fat itself. This mechanism is in contrast to that of uncontrolled thermal injury in which temperatures obtained in deep tissue can reach or exceed 70 degrees C, causing necrotic injury, irregular wound contraction, fat loss, and atrophy, which has been reported with pulsed RF devices. ${ }^{18}$ This study examines the use of the Pellevé system for the application of heat and massage to the periocular area for the treatment of MGD and its related DED (what the authors have dubbed the "ThermaLid procedure").

\section{MATERIALS AND METHODS}

\section{Treatment Device Description}

The Pellevé System was used in conjunction with a neutral plate applied to the patient's back and a sterile, hard, plastic corneal lens protector and a treatment gel, which is applied to the treatment area prior to hand piece use. The electrode is continuously moved over the gel-covered skin, delivering the RF energy where gentle heating is observed. The Pellevé thermometer used to spot-check the surface temperature of the skin was more broadly confirmed to be at the targeted skin surface temperatures by using a FLIR system model T420 infrared camera to measure continuous surface temperatures. 
LipiFlow utilizes a single-use activator, applied to the eyelids, which employs a standardized, computerdirected 12-minute program to raise the temperature of the portion of the eyelids containing the MGs to 42.5 degrees $\mathrm{C}$ and then applies thermal pulsations to that area to eliminate the liquefied MG contents.

\section{Study Population}

Patients were recruited from a private ophthalmology practice in Southern Maine. Ten subjects were enrolled in this prospective, single centre, split-faced study. Candidates who met the inclusion criteria for enrollment in this study were invited to pursue treatment. Patients with any of the subsequent exclusion criteria did not proceed towards treatment (Table 1). A study-specific informed consent form was reviewed and signed to indicate an understanding of all aspects of the trial. The study protocol was approved by the local ethics review committee (UNE IRB Protocol 103114-016). Patients ranged in age from 44 to 90 years old (average age 62.8). Pre-treatment baseline data was collected for all enrolled patients meeting the initial inclusion and exclusion criteria (Table 2).

\section{ThermaLid and Lipiflow Treatment}

A gentle lid debridement was conducted on each lid margin with a "golf club spud" lid debrider prior to determining the treatment eye randomization by virtual coin toss. Subjects received one ThermaLid treatment for one eye and one LipiFlow treatment for the fellow eye. The Pellevé S5 generator was in the "CUT" mode for all treatments. The $10 \mathrm{~mm}$ Pellevé handpiece was used for all treatments. The reusable neutral plate was placed on the subject's back between the shoulders, always ensuring full contact of the plate with the patient's skin. The power settings for the treatment started at 8 and was adjusted to 10 , as tolerated, to optimize heating and as tolerated. It was planned that if the patient showed or verbalized any discomfort during the treatment, the power setting on the PellevéTM S5 generator would be turned down by the user to a comfortable setting for the subject; this proved to be unnecessary for this group of patients. A drop of $0.5 \%$ Proparacaine was used to anesthetize the eye and a Pellevé hard plastic corneal eye shield was used to cover the cornea. Pellevé treatment gel was applied to the skin of the treatment area in a thin even layer. The shielded periocular area of both upper and lower eyelid of the randomized eye was treated. The Pellevé handpiece was placed on the skin with light pressure such that the tip was depressed and activated by pressing the button of the finger switch allowing initiation of the RF current in the Glide-Safe tip. The following protocol was employed:

First Pass: With the activated handpiece in contact with the skin, continuous, small horizontal passes were made with slightly overlapping patterns over the upper or lower lid of one eye.

The surface temperature of the treated skin was continuously assessed using the infrared Pellevé thermometer throughout the procedure. The FLIR model T420 IR camera proved to be superior to the infrared Fluke (standard Pellevé) thermometer and was supplemented for monitoring all cases. When the skin reached a temperature of $38-40^{\circ} \mathrm{C}$, the first "pass" was achieved.

Second Pass: Repeating above steps, however, for the second "pass" the temperature target was $40-42^{\circ} \mathrm{C}$.

Third Pass: Followed the 2nd "pass" by a third 3rd "pass" with a temperature target of $42-43^{\circ} \mathrm{C}$.

Vertical Strokes: Immediately subsequent to this third "pass," the tip was used to provide serial, gentle strokes along the vertical aspect of the tarsus from the base of the tarsus to the lid margin, with slight overlap to massage the lid to express the contents of the MGs in confluent fashion. This meibomian gland expression maneuver, in the context of this heating protocol, constitutes a deviation from a standard, cosmetic-oriented Pellevé procedure and is what the authors have dubbed the ThermaLid procedure. Depending on the size of the lid, the number of slightly overlapping strokes typically varied from 10 to 20 times per pass.

Repeat: Repeat the above step including the meibomian gland expression strokes two additional times (total of 5 "passes" with a total of 3 massages), with the fourth and fifth "passes" targeting $42-44^{\circ} \mathrm{C}$.

Opposing Lid: The opposing lid (lower or upper respectively) of the same eye was then treated in the same manner until the target temperature and massage was achieved.

Opposite Eye: The opposite eye was treated with a standard Lipiflow treatment using a LipiFlow activator and the standard TearScience protocol (the single-use 
TABLE 1 Criteria to Qualify for Study

\section{Inclusion criteria}

- Subject is 18 years or older.

- Willingness and ability to comply with protocol requirements, including returning for follow-up.

- Willingness and ability to provide written informed consent prior to performance of study related procedure.

- Ocular Surface Disease Index (OSDI) score of moderate or more AND Standard Patient Evaluation of Eye Dryness (SPEED) score $\geq 6$.

- TearScience ${ }^{\circledast}$ MGE expression of Moderate or less meibum.

- Wax plugging of MG orifices of few or more.

- TearLab ${ }^{\oplus}$ Tear Osmolarity of 308 or greater OR with an inter-eye difference of 8 mOsm or greater.

- TearScience ${ }^{\circledast}$ Lipiview ICU of 80 or less.

- Oculus Keratograph 5M time-to-first non-invasive tear break-up time (NITBUT) of $\leq 10$ seconds.

- Conjunctival chalasis of grade $2+$ or less.

- Conjunctival and lid margin injection of less than $2+$.

\section{Exclusion Criteria}

- Existing or history of skin disease in the treatment area during the past 1 month.

- History of active collagen vascular disease, or autoimmune disease.

- Subjects who have an implantable pacemaker, automatic implantable defibrillator (AICD), or any other implantable electric device.

- Subjects who have used, within 30 days, any medication that can cause dermal hypersensitivity or affect skin characteristics.

- History of any disease that inhibits pain sensation.

- Concurrent therapy that, in the investigator's opinion, would interfere with the evaluation of the safety or efficacy of the study device.

- Enrollment in any active study involving the use of investigational devices or drugs.

- Meibomian Gland (MG) atrophy in excess of 66\% (as assessed via Oculus Keratograph 5M Meiboscan with less than 33\% remaining MG tissue).

- Schirmer's (5-minute test with topical anesthesia) score $<6 \mathrm{~mm}$ in either eye (indicative of severe Aqueous Tear Deficiency).

- More than $50 \%$ capping of the MGs, Conjunctival chalasis in excess of $2+$.

- Conjunctival or lid margin injection of more than $2+$.

- Presence of superior limbic keratoconjunctivitis (SLK). 
TABLE 2 Pre-treatment Baseline Data for Enrolled Patients

- Informed Consent.

- General demographics and medical history including age and sex at a minimum.

- Subject Self Evaluation Questionnaire (OSDI and SPEED score) for each eye.

- Visual acuity test.

- Oculus Keratograph 5M Non-Invasive Tear film Breakup Time (NITBUT) (Time-to-first TBUT and Average TBUT).

- Tear Meniscus Level (measured with the Oculus Keratograph 5M caliper estimate).

- LipiView average ICU (TearScience $\left.{ }^{\circledast}\right)$.

- MGE grading of MG function (0, minimal, moderate or copious secretions) using the TearScience Korb evaluator(19)

- Marx Line grading(20)

- Tear Osmolarity $\left(\right.$ TearLab $\left.^{\circledast}\right)$.

- Anesthetic 5 minute Schirmer's testing.

- Transillumination and Oculus Meiboscan of lower lids with estimate of remaining MG tissue.

- Grading of wax plugging (scale: none, few, moderate, many).

- Grading of Conjunctival Chalasis (scale: 0-4+).

- Grading of injected lid margin and conjunctiva (grading 0-4+).

- Presence or absence of SLK.

- Lid Wiper epitheliopathy (scale 0-4+).

- Ocular Surface Staining (Lissamine green and Flourescein, grading scale: [scale 0-4+])

activator was applied to the Proparacaine anesthetized eye for a standard, full, 12-minute LipiFlow cycle). Lipiflow treatment for the contralateral eye was followed in accordance with company guidelines.

Pre- to Post-treatment MGD Efficacy Assessment

Grading of the MGs with a meibomian gland evaluator (MGE) (lower lid tested by standard medial, central and lateral pressure of 10 seconds with the TearScience Korb MGE and graded for amount and type of expressed meibum ${ }^{19)}$; TearScience Lipiview average ICU of the tear lipid layer, change in tear breakup time (as assessed by the Oculus Keratograph 5M NITBUT), ocular surface staining pre- to post-treatment, Marx Line grading, Tear Osmolarity, anesthetic Schirmer's testing and ocular surface staining pre- to post-treatment Patient symptoms were assessed by the TearScience SPEED questionnaire and OSDI, per each eye, pre- and post-treatment.

The primary safety endpoint was the composite of device and procedure related adverse events occurring immediately after each treatment and 30 and 90 days after each Pellevé and LipiFlow treatment procedure. A visual acuity test was administered to assess any change in vision. No safety issues were noted at any time point with either technology. 
Subjects received a phone call from the physician's designee one week and one month post treatment to determine if the subject's symptoms had improved. The current SPEED score and OSDI was reviewed and recorded per eye. Subjects returned to the office for a follow-up visit approximately 1 and 3 months post-treatment. Specific assessments were performed at those visits (Table 3).

\section{Statistical Analysis}

The effectiveness of thermaLid and Lipiflow over time was analyzed using Friedman repeated measures 1-way ANOVA tests. Post-hoc comparisons were made by comparing baseline values with the 1 - and 3-month time point measurements using the Dunn's method or the Bonferonni t-tests, as appropriate. The relative effectiveness of thermaLid and Lipiflow ("change" or difference of scores) were compared using the MannWhitney rank sum test or using a Student's t-test, as appropriate. Functional meibomian glands (as assessed by the Korb MGE), OSDI and SPEED questionnaire responses, wax plugs, MARX line, and conjunctival staining were compared between treatment type at the various time points.

\section{RESULTS}

The demographics of the qualified patients are outlined in Table 4.

The Pellevé thermaLid procedure showed parity to a high level of statistical significance across the subjective OSDI and SPEED score criteria of dry eye symptoms as well as improved meibomian gland expression, reduction of wax plugging and conjunctival staining, when compared to the standard Lipiflow procedure in this split-face study (Figures 1-4). The only statistically significant discrepancy between the two procedures was in the MARX Line (ML) group

TABLE 3 Assessment at 1 and 3 Months Post-treatment

- Subject Self Evaluation Questionnaire (OSDI and SPEED score)

- Visual acuity test

- Oculus Keratograph 5M Non-Invasive Tear film Breakup time (NITBUT) (time-to-first TBUT and average TBUT)

- Tear meniscus level (measured with the Oculus Keratograph 5M caliper estimate)

- LipiView average ICU (TearScience).

- MGE grading of MG function (0, minimal, moderate or copious secretions) using the TearScience Korb evaluator

- Marx line grading

- Tear osmolarity $\left(\right.$ TearLab $\left.^{\oplus}\right)$

- Anesthetic 5-minute Schirmer's testing

- Transillumination and Oculus Meiboscan of lower lids with estimate of remaining MG tissue

- Grading of wax plugging (scale: none, few, moderate, many)

- Grading of conjunctival chalasis (scale: $0-4+$ )

- Grading of injected lid margin and conjunctiva (grading 0-4+)

- Presence or absence of SLK

- Lid wiper epitheliopathy (scale $0-4+$ )

- Ocular surface staining (Lissamine green and Flourescein, grading scale: 0-4+)

J Dry Eye Ocu Surf Dis 1(1):e11-e21; March 28, 2018

This article is distributed under the terms of the Creative Commons Attribution-Non

Commercial 4.0 International License. 


\begin{tabular}{lcl}
\hline TABLE 4 Demographics of Qualified Patients & \\
\hline Patient \# & Age/gender & Race \\
\hline 1 & $58 / \mathrm{F}$ & Caucasian \\
2 & $54 / \mathrm{F}$ & Caucasian \\
3 & $56 / \mathrm{F}$ & Caucasian \\
4 & $46 / \mathrm{F}$ & Caucasian \\
5 & $49 / \mathrm{F}$ & Caucasian \\
6 & $92 / \mathrm{F}$ & Caucasian \\
7 & $69 / \mathrm{F}$ & Caucasian \\
8 & $67 / \mathrm{F}$ & Caucasian \\
9 & $55 / \mathrm{M}$ & Caucasian \\
10 & $42 / \mathrm{F}$ & Caucasian \\
\hline
\end{tabular}

(Figure 4). Causes for patient exclusion in the study were also recorded, with the primary reason being an above range interferometric colour unit (ICU) on the LipiView test. (Figure 5).

\section{DISCUSSION}

Lipiflow has been earning a reputation as the gold standard for addressing obstructive MGD, yet is limited by the "one-size-fits-all" activators, the cost of these activators and ancillary equipment and the fact that it can only accomplish the one goal of reducing obstructive MGD. The senior author (EHJ) noted the consistent, positive results achieved by the activators heating the lids to 42.5 degrees Centigrade and applying vectored massage to the meibomian glands to his dry eye patients. The shortcomings of the single size of the activators was demonstrated in several patients with lids too small and tight to accommodate these activators and in some patients where the larger, more lax lids made it difficult to treat the entire lid area with a single treatment. Another issue is that the activators are programmed for a single, 12 minute heating and massaging event, which though consistent in improving obstructive MGD, leaves the impression that a longer or stronger degree of expression might improve some patient outcomes.
Pellevé has been shown to reliably reduce fine lines and wrinkles, tighten loose skin and improve the aesthetic appearance of the face and periorbital area. ${ }^{16}$ The author also noted that the most consistent aesthetic results of the Pellevé system occurred in a similar temperature range. Radiofrequency penetrates deeper and achieves a higher temperature than passive electrical conductive heat. ${ }^{71}$ Applying passes of Pellevé treatment at the slit lamp allowed the author to demonstrate reliable expression of liquid meibum from obstructed glands at pressures approximating the tension in the "GlideSafe ${ }^{\circledR}$ " hand piece. With a contact lens "backstop," the glands could be "milked," precipi-tating an effective meibomian gland expression and purging of inspissated glands that appeared similar to a Lipiflow treatment. Impediments to safely and comfortably treating these glands included the thin-ness of the periorbital skin, the sensitivity of this skin to heat and the propensity to create "hot spots" in the lid, causing an immediate sense of discomfort to the patient and a potential for burns to the lids. When hot spots occur, the patients react by abruptly pulling away, 
FIG. 1 Number of functional meibomian glands. Glands were assessed at baseline (untreated) and at 1 month- and 3-month post-treatment with either thermaLid or Lipiflow. Results for each group are represented as mean \pm SEM. *Values are significantly different from their respective untreated outcome, as determined by the Friedman repeated measures one-way analysis of variance $(\mathrm{p}<0.05) . \mathrm{n}=10$ (all groups).

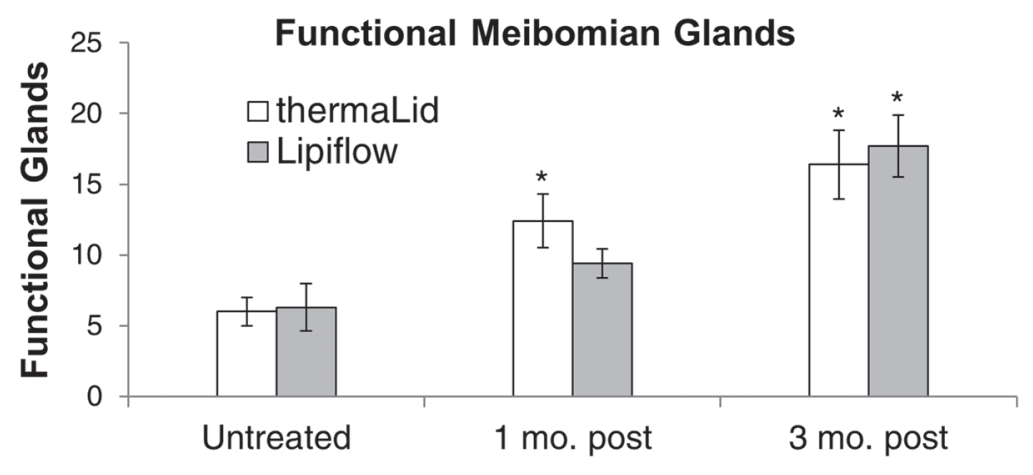

FIG. 2 OSDI and SPEED questionnaires. Subjective assessment of dry eye symptoms using the OSDI and SPEED questionnaires was conducted at baseline (untreated) and at 1 month- and 3-month post-treatment with either thermaLid or Lipiflow. Results for each group are represented as mean \pm SEM. "Values are significantly different from their respective untreated outcome, as determined by the Friedman repeated measures one-way analysis of variance $(\mathrm{p}<0.05) . \mathrm{n}=10$ (all groups).

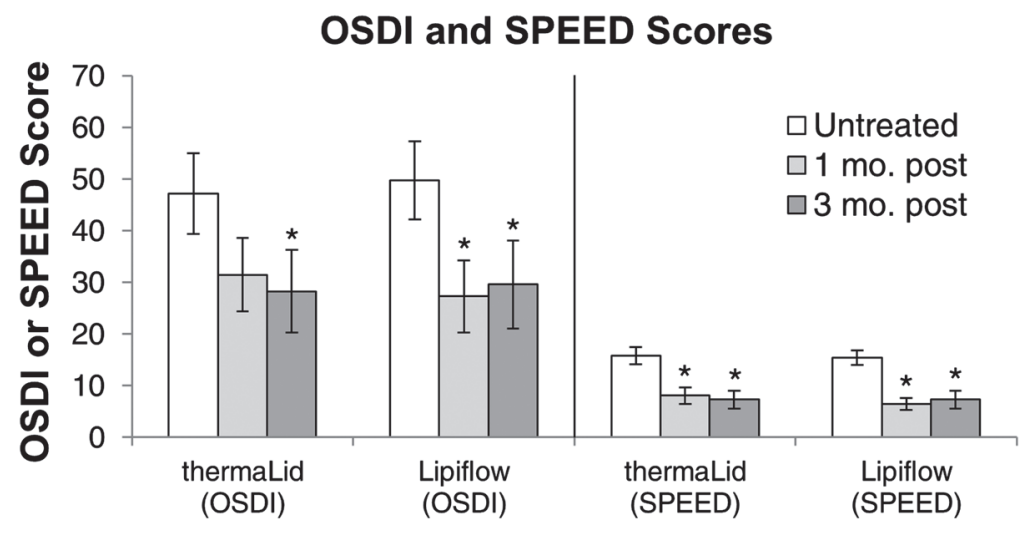

which may leave some areas at a sub-optimal temperature while other areas may be overly heated. The author found that by using a FLIR model T420 IR camera, the entire lid area being treated could also be thermally imaged in colours designating the temperature range, so that a "paint by numbers" heating of the lid could achieve a uniform heating to the desired surface temperature. Slit lamp treatments demonstrated that three consecutive passes at a surface temperature of
42-43 degrees $C$, with immediate vectored massage following each heated pass, allowed reliable expression of meibum from the affected glands. The treatment protocol for this study was therefore modelled after that observational experience.

Of note to this study, the only statistically significant discrepancy between the two procedures was in the MARX Line (ML) group (see Figure 4). Yamaguchi et al found a strong correlation between the ML score and the meibomian gland scores, indicating that the

J Dry Eye Ocu Surf Dis 1(1):e11-e21; March 28, 2018

This article is distributed under the terms of the Creative Commons Attribution-Non

Commercial 4.0 International License. 
FIG. 3 Wax plugs. Assessment of wax plugging was scaled as none (0), few (1), moderate (2) and many (3). Assessment was conducted at baseline (untreated) and at 1 month- and 3-month post-treatment with either ThermaLid or Lipiflow. Results for each group are represented as mean \pm SEM. *Values are significantly different from their respective untreated outcome, as determined by the Friedman repeated measures one-way analysis of variance $(\mathrm{p}<0.05) . \mathrm{n}=10$ (all groups).

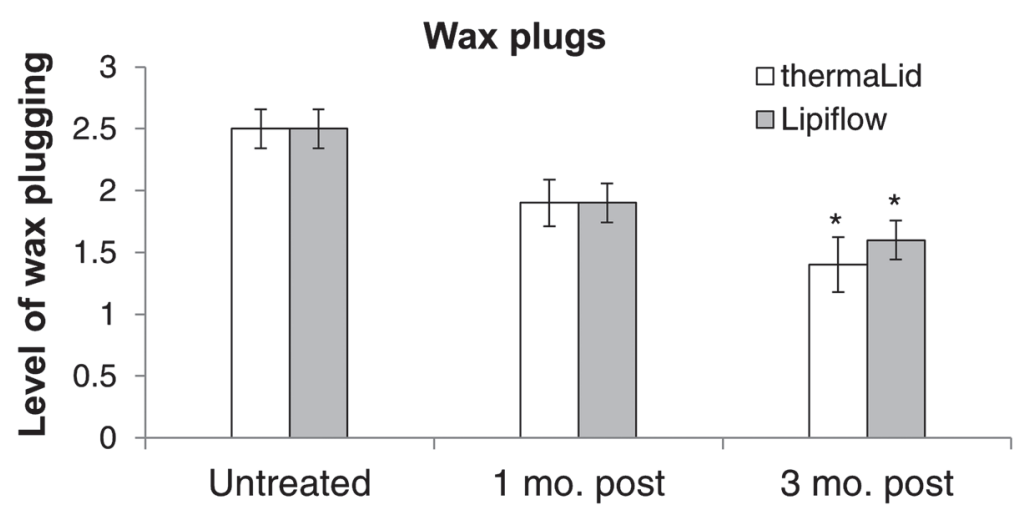

FIG. 4 MARX line (ML) score. ML score was calculated for the outer, middle, and inner thirds of the lower lid for each eye. The scoring scale was $0,1,2$, or 3 , representing increased proximity of the ML to meibomian orifices. Scores were obtained at baseline (untreated) and at 1 month- and 3- month posttreatment with either ThermaLid or Lipiflow. Results for each group are represented as mean \pm SEM. *Values are significantly different from their respective untreated outcome, as determined by the Friedman repeated measures one-way analysis of variance $(p<0.05) . n=10$ (all groups).

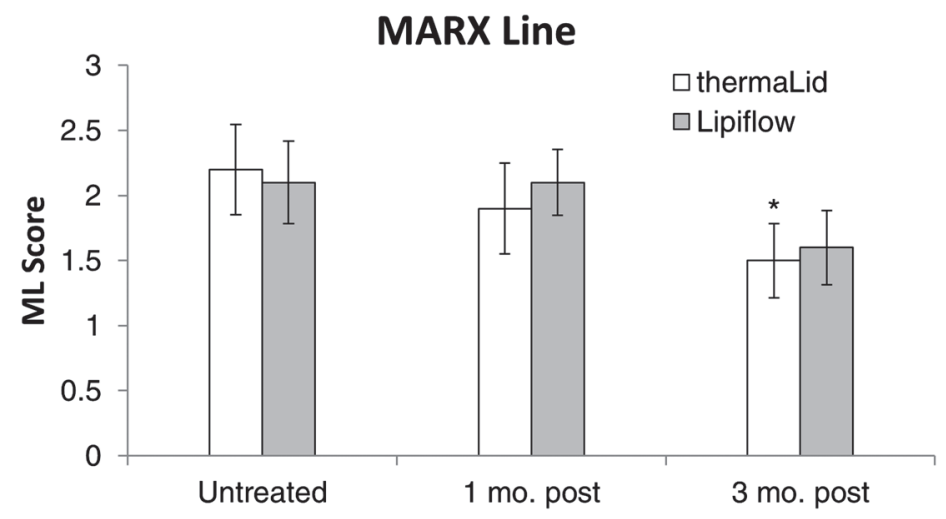

ML score can be used as a simple and rapid screening score for meibomian gland function. ${ }^{20}$ The ability of the ThermaLid procedure to improve ML over Lipiflow, to a statistically significant degree, may indicate superiority in addressing that aspect of MGD over Lipiflow, though the exact mechanism and significance remains uncertain. While this protocol was modelled after Lipiflow heated meibomian gland expression, it is also possible that the customizable nature of this protocol might, with extra passes or expression pres- 
FIG. 5 Causes for exclusion. Patients that did not meet the outlined subjective or objective screening tests were excluded from participation in the study. $n=29$ (all groups).

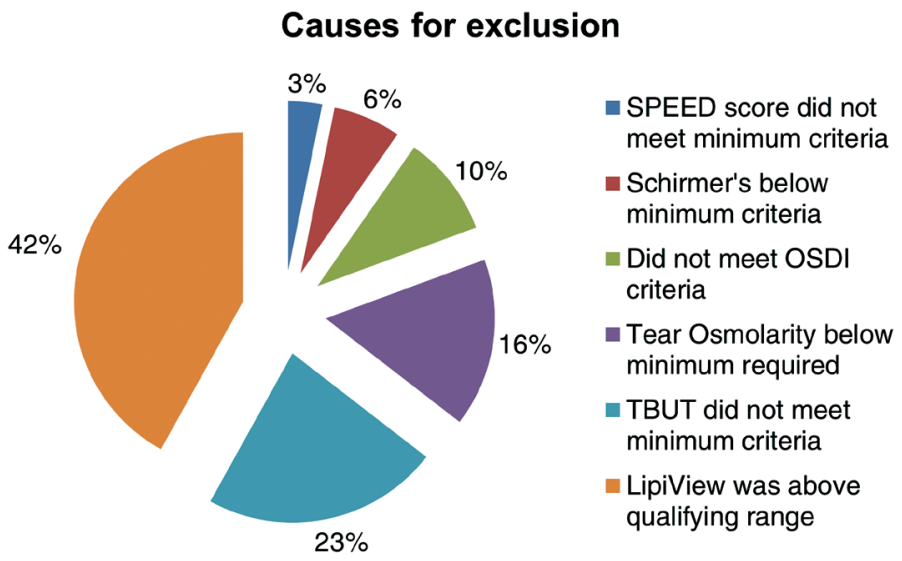

sures, yield superior results over Lipiflow, for some patients. The fact that this study only evaluated ten subjects that were culled from a diverse age group of dry eye sufferers using criteria designed to focus on MGD-related disease leaves the full benefits of eyelid RF treatment yet to become fully elucidated and further studies are warranted.

\section{CONCLUSION}

Obstructive MGD is the most common cause of DED and has been successfully treated with the thermal pulsation system by TearScience (marketed as LipiFlow). This pilot study investigated the potential to use radiofrequency energy for heating, coupled with manual expression, as a means to reduce MGDrelated DED. The initial results from this study appear promising and suggest parity of treatment response compared to Lipiflow within the small subset of patients in this series. Further studies are warranted.

\section{ACKNOWLEDGEMENTS}

Especially helpful to this study were Ian Meng, $\mathrm{PhD}$ (UNE) for his statistical analysis, Edward Bilsky, $\mathrm{PhD}$ (UNE) and Olgun Guvench, PhD (UNE), for their support, the assistance of the UNE IRB, the Maine Technology Institute, which partially helped to fund this research project and Nikita Naumowicz (UNE undergraduate student) for her assistance.

\section{FINANCIAL DISCLOSURE}

The Maine Technology Institute partially funded this project and had no role in the design or conduct of this research. Edward Jaccoma, MD, Craig Lither-land MS, and Andrew Jaccoma, BS, MBA have equal shared interest in eyeThera, a company devoted to the care of DED. Aseef Ahmed, BS, MS, has no potential conflict of interest.

\section{REFERENCES}

1. Reddy P, Grad O, Rajagopalan K. The economic burden of dry eye: a conceptual framework and preliminary assessment. Cornea 2004;23(8):751-61.

2. Lemp MA, Crews LA, Bron AJ, Foulks GN, Sullivan BD. Distribution of aqueous-deficient and evaporative dry eye in a clinic-based patient cohort: a retrospective study. Cornea 2012;31(5):472-8.

3. Tong L, Chaurasia SS, Mehta JS, Beuerman RW. Screening for meibomian gland disease: its relation to dry eye subtypes and symptoms in a tertiary referral clinic in singapore. Invest Ophthalmol Vis Sci 2010;51(7):3449-54.

4. Viso E, Rodriguez-Ares MT, Gude F. Prevalence of and associated factors for dry eye in a Spanish adult population (the Salnes Eye Study). Ophthalmic Epidemiol 2009;16(1):15-21.

5. Stapleton F, Garrett Q, Chan C, Craig JP. Dry eye: A practical approach. Chan C, editor. Berlin, Germany: Springer-Verlag Berlin Heidelberg; 2015. 
6. Moss SE, Klein R, Klein BE. Prevalence of and risk factors for dry eye syndrome. Arch Ophthalmol 2000;118(9):1264-8.

7. Blackie CA, Solomon JD, Greiner JV, Holmes M, Korb DR. Inner eyelid surface temperature as a func-tion of warm compress methodology. Optom Vis Sci 2008;85(8):675-83.

8. Buchholz P, Steeds CS, Stern LS, Wiederkehr DP, Doyle JJ, Katz LM, et al. Utility assessment to measure the impact of dry eye disease. Ocul Surf 2006;4(3):155-61

9. Chan C. Dry Eye: A Practical Approach. Chan C, editor. Berlin, Germany: Springer-Verlag Berlin Heidelberg; 2015.

10. Geerling G, Baudouin C, Aragona P, Rolando M, Boboridis KG, Benitez-Del-Castillo JM, et al. Emerging strategies for the diagnosis and treatment of meibomian gland dysfunction: Proceedings of the OCEAN group meeting. Ocul Surf 2017;15(2):179-92.

11. Baudouin C, Messmer EM, Aragona P, Geerling G, Akova YA, Benitez-del-Castillo J, et al. Revisiting the vicious circle of dry eye disease: a focus on the pathophysiology of meibomian gland dysfunction. $\mathrm{Br}$ J Ophthalmol 2016;100(3):300-6.

12. Blackie CA, Carlson AN, Korb DR. Treatment for meibomian gland dysfunction and dry eye symptoms with a single-dose vectored thermal pulsation: a review. Curr Opin Ophthalmol 2015;26(4):306-13.

13. Rusciani A, Curinga G, Menichini G, Alfano C, Rusciani L. Nonsurgical tightening of skin laxity: a new radiofrequency approach. J Drugs Dermatol 2007;6(4):381-6.
14. Taub AF, Tucker RD, Palange A. Facial tightening with an advanced 4-MHz monopolar radiofrequency device. J Drugs Dermatol 2012;11(11):1288-94.

15. Stampar M. The Pelleve procedure: an effective method for facial wrinkle reduction and skin tightening. Facial Plast Surg Clin North Am 2011;19(2):335-45.

16. Javate RM, Cruz RT, Jr., Khan J, Trakos N, Gordon RE. Nonablative 4-MHz dual radiofrequency wand rejuvenation treatment for periorbital rhytides and midface laxity. Ophthal Plast Reconstr Surg 2011;27(3):180-5.

17. Zelickson BD, Kist D, Bernstein E, Brown DB, Ksenzenko S, Burns J, et al. Histological and ultrastructural evaluation of the effects of a radiofrequency-based nonablative dermal remodeling device: a pilot study. Arch Dermatol 2004;140(2):204-9.

18. Fitzpatrick R, Geronemus R, Goldberg D, Kaminer M, Kilmer S, Ruiz-Esparza J. Multicenter study of noninvasive radiofrequency for periorbital tissue tightening. Lasers Surg Med 2003;33(4):232-42.

19. Korb DR, Blackie CA. Meibomian gland diagnostic expressibility: correlation with dry eye symptoms and gland location. Cornea 2008;27(10):1142-7.

20. Yamaguchi M, Kutsuna M, Uno T, Zheng X, Kodama T, Ohashi Y. Marx line: fluorescein staining line on the inner lid as indicator of meibomian gland function. Am J Ophthalmol 2006;141(4):669-75. 